\title{
Prognostic relevance of FGFR2 expression in stage II/III gastric cancer with curative resection and S-1 chemotherapy
}

\author{
KEI HOSODA, KEISHI YAMASHITA, HIDEKI USHIKU, AKIRA EMA, \\ HIROMITSU MORIYA, HIROAKI MIENO, MARIE WASHIO and MASAHIKO WATANABE
}

Department of Surgery, Kitasato University School of Medicine, Sagamihara, Kanagawa 252-0374, Japan

Received August 21, 2017; Accepted November 22, 2017

DOI: $10.3892 / \mathrm{ol} .2017 .7515$

\begin{abstract}
Curative gastrectomy and adjuvant chemotherapy using S-1 is a standard treatment for stage II/III gastric cancer in Japan. The purpose of the present study was to evaluate the prognostic relevance of fibroblast growth factor receptor (FGFR)2 expression in patients with stage II/III gastric cancer that underwent postoperative adjuvant chemotherapy with S-1. Formalin-fixed paraffin-embedded surgical specimens were retrospectively examined in 167 patients with stage II/III gastric cancer that underwent curative gastrectomy followed by adjuvant S1 chemotherapy. FGFR2 expression was measured using immunohistochemistry (IHC) staining. The IHC results for FGFR2 were as follows: Grade 1+, 32; grade 2+, 80; grade 3+, 55 patients. The FGFR2 expression level was not significantly associated with relapse-free or overall survival rates. However, in the diffuse type, the FGFR2 expression level tended to be negatively correlated with relapse-free survival. In particular, the proportion of patients who recurred $>5$ years following surgery was significantly larger in the FGFR 2 grade $3+$ group than in the grade $1+, 2+$ group $(4 / 22$ vs. $1 / 35 ; \mathrm{P}=0.047)$. The recurrent sites of long-term failure were mostly peritoneum among the diffuse type. To the best of our knowledge, the present study indicated for the first time that FGFR2 could predict long-term failure of adjuvant S-1 chemotherapy in curative advanced gastric cancer. There was no interaction between FGFR2 expression and patient survival outcomes in stage II/III gastric cancer. Patients with FGFR2 3+ in stage II/III gastric cancer should carefully be followed-up for $>5$ years after surgery.
\end{abstract}

Correspondence to: Dr Kei Hosoda, Department of Surgery, Kitasato University School of Medicine, 1-15-1 Kitasato, Minami-ku, Sagamihara, Kanagawa 252-0374, Japan

E-mail: k.hosoda@kitasato-u.ac.jp

Key words: FGFR2, gastric cancer, recurrence timing, S-1, survival outcomes

\section{Introduction}

Gastric cancer is one of the leading causes of cancer related death worldwide (1). Advanced gastric cancer exhibits poor prognosis, and even optimal combination modalities of surgery, chemotherapy, and radiotherapy cannot attain satisfactory survival outcomes.

S-1 is a fluoropyrimidine containing compound consisting of a combination of tegafur, gimeracil, and oteracil potassium. A randomized phase III trial of adjuvant chemotherapy with S-1 (ACTS-GC) demonstrated that surgery with S-1 adjuvant chemotherapy surpassed surgery alone in stage II/III gastric cancer $(2,3)$. Thereafter, curative gastrectomy and adjuvant chemotherapy using S-1 have become a standard treatment for stage II/III gastric cancer in Japan. However, in the subgroup analysis of ACTS-GC, the 5-year overall survival (OS) rate of patients with stage III disease was $50.2 \%$, leaving room for improvement.

Molecular targeted therapy is an alternative therapeutic tool for advanced cancer, and the receptor tyrosine kinase (RTK) family is one such promising candidate target. The fibroblast growth factor (FGF) family which includes important regulatory factors of cell growth and differentiation, has been found to be involved in embryonic development, angiogenesis, and tumorigenesis $(4,5)$. The FGF receptor (FGFR) family, one type of RTK family, is a transmembrane tyrosine kinase receptor involved in signaling via interaction with the FGF family. To date, four different members of the FGFR family, FGFR1, FGFR2, FGFR3 and FGFR4, have been cloned and characterized (6-8). FGFR2 gene amplification was initially found in a gastric cancer cell line originating from diffuse type gastric cancer (9). FGFR2 has been demonstrated to be a poor prognostic biomarker $(10,11)$ and antibodies $(12-14)$ or small molecule inhibitors (15-18) targeting FGFR2 can suppress gastric cancer progression in vivo and in vitro.

The human epidermal growth factor receptor (EGFR/HER) family is involved in complex and tightly controlled signaling pathways that regulate various cellular functions, such as cell proliferation, organ development, and organ repair. Among four members of the HER family (EGFR, HER2, HER3 and HER4), HER3 is indicated to play an important role in HER signaling and drug resistance (19). With dimerization with EGFR or HER2, HER3 signals through phosphatidylinositol 3-kinase pathway $(20,21)$. 
We have previously analyzed various RTK expressions and reported that HER3 and EGFR were significant and marginally significant independent prognostic factors, respectively, in patients with stage II/III gastric cancer who underwent curative gastrectomy followed by adjuvant chemotherapy with S-1 (22). However, we have not included this critical RTK of FGFR2 in the study. We hypothesized that FGFR2 alone or that in combination with HER3 and EGFR plays an important role on survival outcomes of these patients. Therefore, we analyzed correlation between HER3 or EGFR expression and FGFR2 expression using the identical patients of the previous study, and evaluated the prognostic impact of FGFR2 expression in patients with stage II/III gastric cancer treated by curative gastrectomy followed by adjuvant chemotherapy with $\mathrm{S}-1$.

\section{Materials and methods}

Patients. From January 2000 to December 2010, 172 patients with stage II/III advanced gastric cancer underwent curative surgery followed by adjuvant S-1 chemotherapy at Kitasato University Hospital. Of the 172 patients, informed consent to use specimens was obtained from 167 patients, for whom the median follow up term was 75 months (inter quartile range, 61-90 months). These patients were identical to the patients whom we had analyzed previously (22).

This study was conducted in accordance with the Declaration of Helsinki and was approved by the Research Ethics Committee of the Kitasato University School of Medicine (Sagamihara, Japan).

Surgery. Gastrectomy with D1 lymph node dissection was performed for clinical stage IA gastric cancer $(n=25)$. Gastrectomy with D2 lymph node dissection was otherwise performed $(n=142)$. The extent of lymph node dissection was determined according to the Japanese Classification of Gastric Carcinoma (second English edition) (23).

Postoperative chemotherapy of $S-1$. The dose of S-1 was based on body-surface area: $<1.25 \mathrm{~m}^{2}$ (80 $\mathrm{mg}$ daily); $\geq 1.25 \mathrm{~m}^{2}$ but $<1.50 \mathrm{~m}^{2}$ (100 mg daily); $\geq 1.50 \mathrm{~m}^{2}$ (120 mg daily). The adjuvant S-1 was administered for 4 weeks followed by 2 weeks rest. This 6 -week cycle was repeated principally during the first year after surgery.

Immunohistochemistry (IHC) analysis. Tumor specimens used in this study were derived from routine formalin-fixed, paraffin embedded tissue samples obtained from resected gastric cancer specimens. Sections (3- $\mu \mathrm{m}$ thick) were cut from the paraffin blocks and mounted on silanized slides. For IHC analysis of FGFR2, tumor tissue sections were deparaffinized in xylene and dehydrated with graded ethanol. After washing with distilled water, antigen unmasking was performed with a pressure cooker using citrate buffer. Endogenous peroxidase activity was blocked by incubation in $3 \% \mathrm{H}_{2} \mathrm{O}_{2} /$ methanol for $15 \mathrm{~min}$, and nonspecific antibody binding was blocked by incubation with $1 \%$ diluted normal horse serum for $30 \mathrm{~min}$. Sections were then incubated at room temperature for $60 \mathrm{~min}$ with the following antibodies: Mouse FGFR2 monoclonal antibody (M01, clone 1G3) (dilution of 1:800; Abnova, Heidelberg, Germany, ). Immune complexes were detected with a Vectastain Elite ABC kit (Vector Laboratories, Inc., Burlingame, CA, USA) according to the manufacturer's instructions. These immune complexes were detected using the 3,3'-diaminobenzidine tetrahydrochloride substrate with/without nickel ammonium sulfate (DAB; Vector Laboratories, Inc.) as a chromogen for $5 \mathrm{~min}$. Sections were counterstained with hematoxylin.

IHC staining of HER3 and EGFR was performed as previously reported (22).

Scoring system of IHC staining. To assess IHC staining, all slides were observed under a microscope. Investigators were blinded to the prognostic analysis data. We adopted almost the same scoring system for FGFR2 as was reported by Nagatsuma et al (24). We defined the scores for FGFR2 as follows: 0 , staining reactivity in $<50 \%$ of cancer cells; $1+$, cytoplasm and/or nuclear reactivity with faint staining in more than $50 \%$ of the cancer cells; $2+$, cytoplasm and/or nuclear reactivity with weak or moderate staining in more than $50 \%$ of the cancer cells; $3+$, cytoplasm and/or nuclear reactivity with strong staining in more than $50 \%$ of the cancer cells.

The scoring system for IHC staining of HER3 and EGFR was described before (22).

Statistical analysis. Relapse-free survivals (RFS) were measured from the dates of surgery to the dates of relapse of gastric cancers or dates of last follow-up. Patients who died from causes other than gastric cancer were regarded as censored at the time of death. Patients who were alive at the times of their last visits were also regarded as censored. Student's t-test was used to analyze continuous variables, and Chi square test or Fisher's exact test was used to analyze categorical variables. Survival curves were estimated using the Kaplan-Meier method, and the statistical significance of differences between survival curves was assessed using the log-rank test. Survival analyses were conducted using a Cox proportional hazards model. All calculations were performed using the JMP ${ }^{\circledR} 11.2$ (SAS Institute Inc., Cary, NC, USA), and $\mathrm{P}<0.05$ was considered to indicate a statistically significant difference.

\section{Results}

Patient characteristics and stage distributions. The characteristics of patients included in this study are listed in Table I. More than half of the patients had pT4a tumors. The FGFR2 IHC results were obtained in all 167 specimens as follows: FGFR2 grade 1+, 32 (19\%); FGFR2 grade 2+, 80 (48\%); FGFR2 grade $3+, 55(33 \%)$. Representative examples of immunostaining for FGFR2 in diffuse type cancer (Fig. 1A) and intestinal type cancer (Fig. 1B).

Relations of FGFR2 expression to clinicopathological features and correlation between FGFR2 expression and HER3 or EGFR expression. We divided the study samples into two groups [low FGFR2 expression group: FGFR2 IHC grade 1+, 2+ ( $\mathrm{n}=112)$; high FGFR2 expression group: FGFR2 IHC grade $3+(n=55)]$. The relations of IHC staining levels 
Table I. Characteristics of the patients.

\begin{tabular}{|c|c|}
\hline Category & $n=167$ \\
\hline \multicolumn{2}{|l|}{ Age (years) } \\
\hline Median (range) & $65(35-83)$ \\
\hline \multicolumn{2}{|l|}{$\operatorname{Sex}, \mathrm{n}(\%)$} \\
\hline Male & $117(70)$ \\
\hline \multicolumn{2}{|c|}{ Tumor stage (TNM 7th), n (\%) } \\
\hline $\mathrm{T} 2$ & $28(17)$ \\
\hline $\mathrm{T} 3$ & $34(20)$ \\
\hline $\mathrm{T} 4 \mathrm{a}$ & $103(62)$ \\
\hline $\mathrm{T} 4 \mathrm{~b}$ & $2(1)$ \\
\hline \multicolumn{2}{|c|}{ Nodal stage (TNM 7th), n (\%) } \\
\hline No & $24(14)$ \\
\hline N1 & $43(26)$ \\
\hline N2 & $38(23)$ \\
\hline N3 & $62(37)$ \\
\hline \multicolumn{2}{|c|}{ TNM 7th stage, n (\%) } \\
\hline IIA & $13(8)$ \\
\hline IIB & $36(22)$ \\
\hline IIIA & $43(26)$ \\
\hline IIIB & $37(22)$ \\
\hline IIIC & $38(23)$ \\
\hline \multicolumn{2}{|c|}{ Histological type, n (\%) } \\
\hline Diffuse & $58(35)$ \\
\hline Intestinal & $109(65)$ \\
\hline \multicolumn{2}{|c|}{ FGFR2 IHC status, n (\%) } \\
\hline 0 & 0 \\
\hline $1+$ & $32(19)$ \\
\hline $2+$ & $80(48)$ \\
\hline $3+$ & $55(33)$ \\
\hline \multicolumn{2}{|c|}{ HER3 IHC status, n (\%) } \\
\hline 0 & $69(41)$ \\
\hline $1+$ & $68(41)$ \\
\hline $2+$ & $30(18)$ \\
\hline $3+$ & 0 \\
\hline \multicolumn{2}{|c|}{ EGFR IHC status, n (\%) } \\
\hline 0 & 0 \\
\hline $1+$ & $62(37)$ \\
\hline $2+$ & $57(34)$ \\
\hline $3+$ & $48(29)$ \\
\hline
\end{tabular}

TNM, tumor node metastasis; IHC, immunohistochemistry; EGFR, epidermal growth factor receptor; HER3, human epidermal growth factor receptor 3 .

to clinicopathological features were then examined. FGFR2 IHC staining level was significantly related to patients' age $(\mathrm{P}=0.043)$ and tended to be related to lymph node metastasis $(\mathrm{P}=0.099)$ (Table II). There was no correlation between FGFR2 expression and HER3 expression, whereas the FGFR2 expression level was correlated with the EGFR expression level ( $\mathrm{P}=0.037$; Fig. 2).
Table II. Relations of FGFR2 IHC staining levels to clinicopathological features.

\begin{tabular}{|c|c|c|c|}
\hline Variable/category & $\begin{array}{c}\text { FGFR2 } \\
1+/ 2+(n=112)\end{array}$ & $\begin{array}{c}\text { FGFR2 } \\
3+(n=55)\end{array}$ & P-value \\
\hline Age & & & 0.043 \\
\hline$<65$ & 53 & 17 & \\
\hline$\geq 65$ & 59 & 38 & \\
\hline Sex & & & 1.000 \\
\hline Male & 78 & 39 & \\
\hline Female & 34 & 16 & \\
\hline Serosal invasion & & & 0.734 \\
\hline Absent & 43 & 19 & \\
\hline Present & 69 & 36 & \\
\hline Lymph node metastasis & & & 0.099 \\
\hline Absent & 20 & 4 & \\
\hline Present & 92 & 51 & \\
\hline Cancer stage, n (\%) & & & 0.283 \\
\hline IIA/IIB & 36 & 13 & \\
\hline IIIA/IIIB/IIIC & 76 & 42 & \\
\hline Histologic type, n (\%) & & & 0.604 \\
\hline Diffuse & 37 & 21 & \\
\hline Intestinal & 75 & 34 & \\
\hline
\end{tabular}

IHC, immunohistochemistry; FGFR, fibroblast growth factor receptor.

Effects of clinicopathological factors and FGFR2 expression on RFS and OS. Five-year RFS and OS were 67.7 and 70.1\%, respectively. Elderly patients aged 65 or more had significantly worse RFS and OS than young patients aged less than 65 [hazard ratio (HR) 1.75, 95\% confidence interval (CI) 1.02-3.10, $\mathrm{P}=0.042$ and HR $2.2095 \%$ CI 1.28-3.94, $\mathrm{P}=0.005$ ], while female patients had significantly better OS than male patients (HR 0.47, 95\% CI 0.24-0.85, $\mathrm{P}=0.012$ ). Patients with TNM stage III had significantly worse RFS than those with TNM stage II (HR 2.68, 95\% CI 1.38-5.87, $\mathrm{P}=0.003$ ). On the other hand, there was no relation of FGFR2 expression status to RFS or OS in the study group (Table III, Fig. 3). However, when we restricted patients to those with diffuse type cancer, patients with high FGFR2 expression had slightly worse RFS than those with low FGFR2 expression ( $\mathrm{P}=0.37$; Fig. 3C). Moreover, when we combined FGFR2 and HER3 expression, prognostic impact of HER3 was augmented by FGFR2 expression with 5-year survival rate of 95 and $51 \%$ in patients with FGFR2/HER3 3+/0 and FGFR2/HER3 3+/1+ or 2+, respectively $(\mathrm{P}<0.001$; Fig. 4A). Statistical significant differences were also found between the FGFR2/HER3 3+/0 group and the FGFR2/HER3 $1+$ or $2+/ 1+$ or $2+$ group and between the FGFR2/HER3 1+ or 2+/0 group and the FGFR2/HER3 3+/1+ or $2+$ group with $\mathrm{P}$ values of 0.021 and 0.004 , respectively. When we combined FGFR2 and EGFR expression, no correlation was found in RFS (Fig. 4B).

We further analyzed RFS using the combined expressions of the three biomarkers (Fig. 5). Patients with FGFR2 3+, HER3 $1+/ 2+$, and EGFR $3+$ belonged to one of the worst 

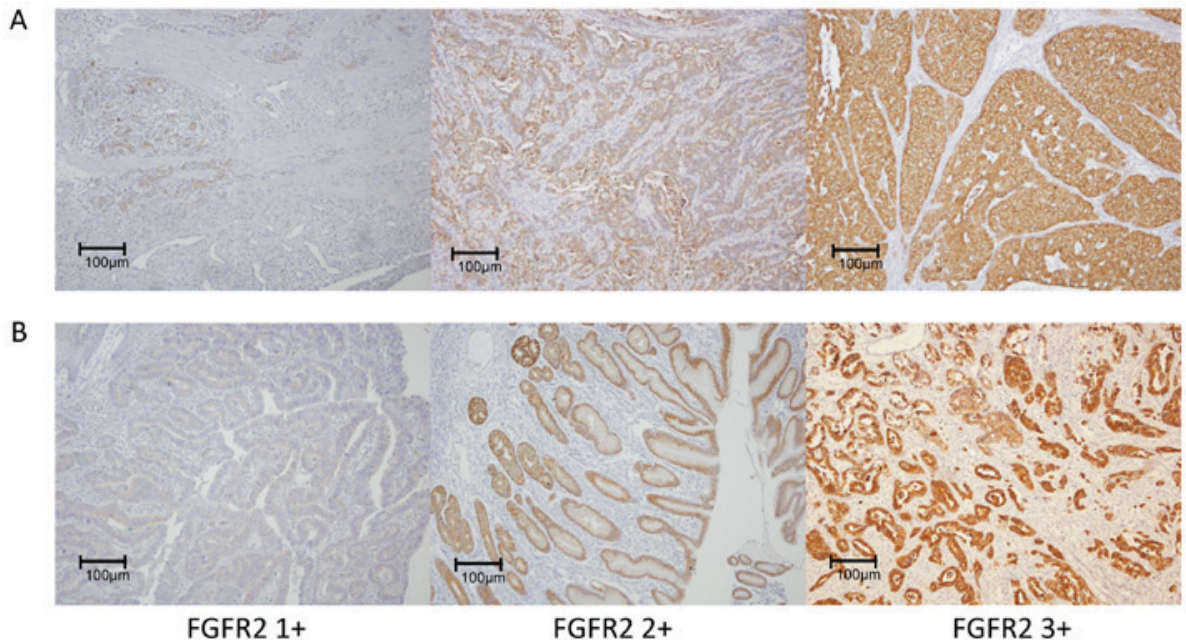

Figure 1. Immunohistochemical staining of FGFR2 expression in the stage II/III gastric cancer of (A) diffuse type and (B) intestinal type (magnification, $\mathrm{x} 100)$. FGFR, fibroblast growth factor receptor.

\begin{tabular}{|c|c|c|c|c|}
\hline \multicolumn{2}{|c|}{} & \multicolumn{3}{|c|}{ HER3 } \\
\cline { 3 - 5 } \multicolumn{2}{c|}{} & $2+$ & $1+$ & 0 \\
\hline \multirow{3}{*}{ FGFR2 } & $3+$ & 9 & 26 & 20 \\
\cline { 2 - 5 } & $2+$ & 16 & 29 & 35 \\
\cline { 2 - 5 } & $1+$ & 5 & 13 & 14 \\
\hline
\end{tabular}

$P=0.77$

\begin{tabular}{|c|c|c|c|c|}
\hline \multicolumn{2}{|c|}{} & \multicolumn{3}{|c|}{ EGFR } \\
\cline { 3 - 6 } \multicolumn{2}{|c|}{} & $3+$ & $2+$ & $1+$ \\
\hline \multirow{3}{*}{ FGFR2 } & $3+$ & 19 & 17 & 19 \\
\cline { 2 - 5 } & $2+$ & 15 & 30 & 35 \\
\cline { 2 - 5 } & $1+$ & 14 & 10 & 8 \\
\hline
\end{tabular}

$\mathrm{P}=0.068$

\begin{tabular}{|c|c|c|c|}
\hline \multicolumn{2}{|c|}{} & \multicolumn{2}{c|}{ HER3 } \\
\cline { 3 - 4 } \multicolumn{2}{|c|}{} & $2+/ 1+$ & 0 \\
\hline \multirow{2}{*}{ FGFR2 } & $3+/ 2+$ & 80 & 55 \\
\cline { 2 - 4 } & $1+$ & 18 & 14 \\
\hline
\end{tabular}

$P=0.84$

\begin{tabular}{|c|c|c|c|}
\hline \multicolumn{2}{|c|}{} & \multicolumn{2}{c|}{ EGFR } \\
\cline { 3 - 4 } \multicolumn{2}{|c|}{} & $3+$ & $2+/ 1+$ \\
\hline \multirow{3}{*}{ FGFR2 } & $3+/ 2+$ & 34 & 101 \\
\cline { 2 - 4 } & $1+$ & 14 & 18 \\
\hline
\end{tabular}

$\mathrm{P}=0.037$

Figure 2. Correlation between FGFR2 expression and HER3 or EGFR expression. There is no correlation between FGFR2 expression and HER3 expression, whereas the FGFR2 expression level was correlated with the EGFR expression level. FGFR, fibroblast growth factor receptor; HER, human epidermal growth factor receptor; EGFR, EGFR, epidermal growth factor receptor.

survival groups with 5 year relapse-free and OS rates of 53 and $59 \%$, respectively. However, no significant difference was found between the FGFR2 3+, HER3 1+/2+, EGFR 3+ group, the FGFR2 1+, 2+, HER3 1+/2+, EGFR 3+ group, and the FGFR2 3+, HER3 1+/2+, EGFR 1+, 2+ group $(\mathrm{P}=0.82)$.

Timing of recurrence. Of the 165 patients, 57 recurred. Proportion or patients with recurrence was similar between the high FGFR2 expression group and the low FGFR2 expression group [40\% (22/55) vs. $31 \%(35 / 112), \mathrm{P}=0.27]$. Of the 57 recurred patients, 5 recurred more than 5 years after surgery (Table IV). Proportion of patients who recurred more than 5 years after surgery was significantly larger in the high FGFR2 expression group than in the low FGFR2 expression group [18\% (4/22) vs. $2.9 \%$ (1/35), $\mathrm{P}=0.047]$ (Fig. 6). All the 5 patients had tumors of diffuse type and serosal invasion (pT4a). Reciprocal relation was found between FGFR2 expression and EGFR expression. That is, 4 had tumors with FGFR2 grade $3+$ and EGFR grade 1+, while the other had tumors with FGFR2 grade $1+$ and EGFR grade $3+$. One female patient with high FGFR2 expression recurred more than 10 years after surgery. She was 55 years old at the time of surgery and underwent total gastrectomy with D2 lymph node dissection. She had a tumor with serosal invasion (pT4a) but no lymph node metastasis at the time of surgery. At the 10th year check-up, CT scan revealed that she had para-aortic lymph node metastasis.

\section{Discussion}

The present study retrospectively evaluated the influence of FGFR2 expression on the survival outcomes of patients with stage II/III gastric cancer who underwent curative gastrectomy followed by adjuvant chemotherapy using S1. The FGFR2 
Table III. Cox proportional hazards analysis of clinicopathological factors for OS and RFS.

\begin{tabular}{|c|c|c|c|c|c|c|c|c|}
\hline \multirow[b]{2}{*}{ Variable } & \multirow[b]{2}{*}{ Category } & \multirow[b]{2}{*}{$\begin{array}{l}\text { No. of } \\
\text { patients }\end{array}$} & \multicolumn{3}{|c|}{ RFS } & \multicolumn{3}{|c|}{ OS } \\
\hline & & & $\begin{array}{c}\text { 5-year } \\
\text { survival }(\%)\end{array}$ & $\begin{array}{c}\text { HR } \\
(95 \% \mathrm{CI})\end{array}$ & P-value & $\begin{array}{c}\text { 5-year } \\
\text { survival }(\%)\end{array}$ & $\begin{array}{c}\mathrm{HR} \\
(95 \% \mathrm{CI})\end{array}$ & P-value \\
\hline \multirow[t]{2}{*}{ Age } & $<65$ & 70 & 76.6 & 1.0 & 0.042 & 83.1 & 1.0 & 0.005 \\
\hline & $\geq 65$ & 97 & 60.8 & $1.75(1.02-3.10)$ & & 60.9 & $2.20(1.28-3.94)$ & \\
\hline \multirow[t]{2}{*}{ Sex } & Male & 117 & 62.5 & 1.0 & 0.084 & 62.9 & 1.0 & 0.012 \\
\hline & Female & 50 & 79.5 & $0.59(0.31-1.07)$ & & 85.8 & $0.47(0.24-0.85)$ & \\
\hline \multirow[t]{2}{*}{ TNM stage } & IIA/IIB & 49 & 84.5 & 1.0 & 0.003 & 78.3 & 1.0 & 0.088 \\
\hline & IIIA/IIIB/IIIC & 118 & 60.8 & $2.68(1.38-5.87)$ & & 66.7 & $1.67(0.93-3.22)$ & \\
\hline \multirow[t]{2}{*}{ Histologic type } & Differentiated & 58 & 70.4 & 1.0 & 0.410 & 61.9 & 1.0 & 0.096 \\
\hline & Undifferentiated & 109 & 62.4 & $0.80(0.47-1.38)$ & & 74.6 & $0.64(0.38-1.09)$ & \\
\hline \multirow[t]{2}{*}{ FGFR2 IHC status } & $1+, 2+$ & 112 & 68.1 & 1.0 & 0.377 & 71.2 & 1.0 & 0.411 \\
\hline & $3+$ & 55 & 66.6 & $1.27(0.74-2.16)$ & & 67.8 & $1.25(0.73-2.09)$ & \\
\hline
\end{tabular}

RFS, relapse-free survival; OS, overall survival; HR, hazard ratio; CI, confidence interval; TNM, tumor node metastasis; FGFR, fibroblast growth factor receptor; IHC, immunohistochemistry.
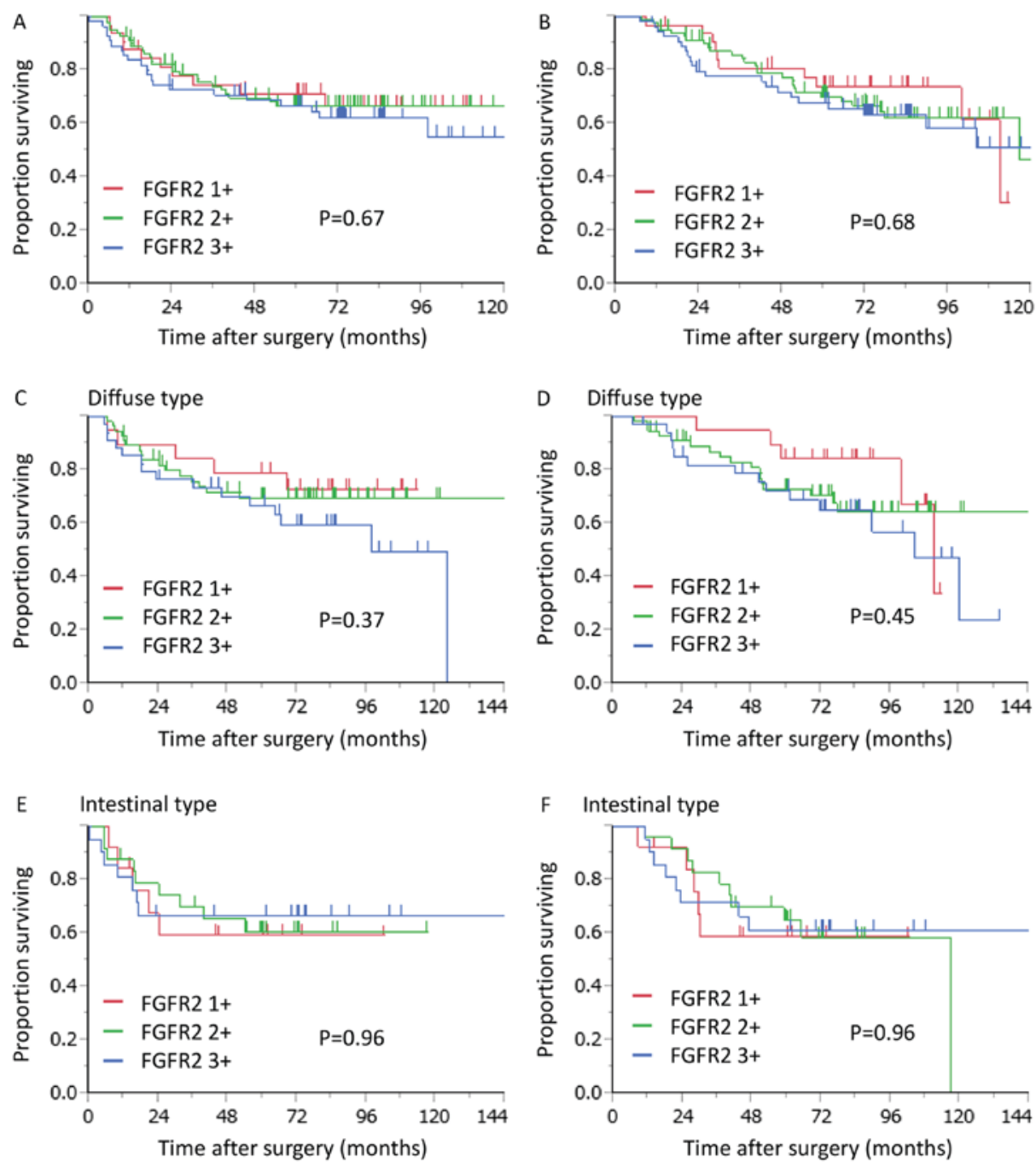

Figure 3. Kaplan-Meier curves for (A) relapse-free survival and (B) overall survival in patients with stage II/III gastric cancer stratified by the FGFR2 IHC staining levels. There is no relation of FGFR2 expression status to relapse-free survival or overall survival. Kaplan-Meier curves for (C and E) relapse-free survival and (D and F) overall survival in patients with diffuse type, intestinal type stage II/III gastric cancer stratified by the FGFR2 IHC staining levels. In patients with diffuse type cancer, the FGFR2 expression tended to be negatively correlated to (C) relapse-free survival ( $\mathrm{P}=0.37$ ). FGFR, fibroblast growth factor receptor; IHC, immunohistochemistry. 

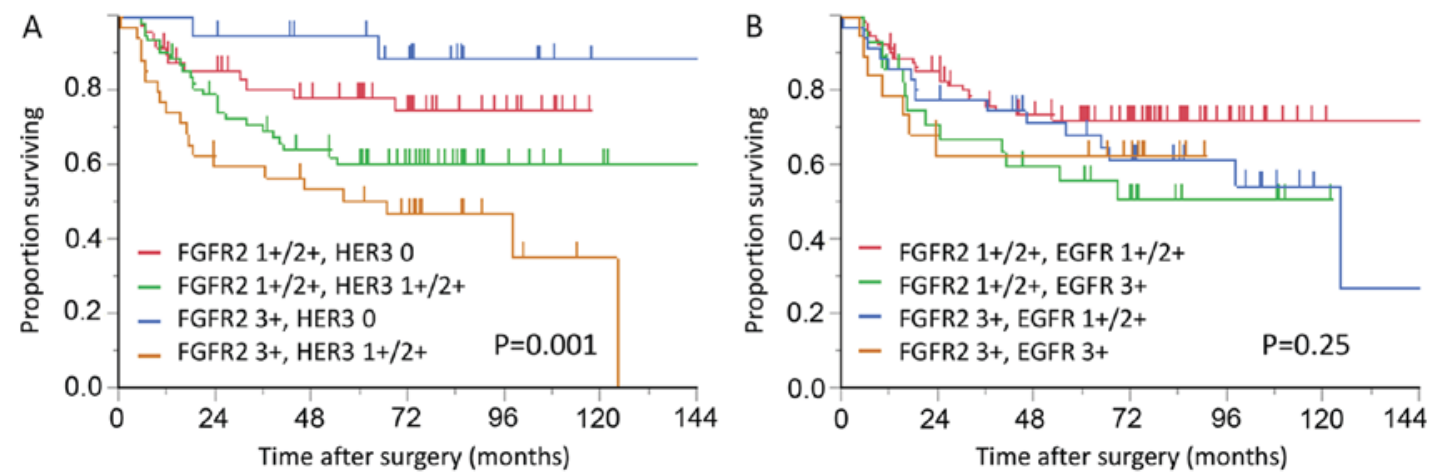

Figure 4. Kaplan-Meier curves for relapse-free survival in patients with stage II/III gastric cancer stratified by combination of (A) FGFR2 and HER3 expressions, and (B) FGFR2 and EGFR expressions. Prognostic impact of HER3 is augmented by FGFR2 expression with 5-year survival rate of 78 and $51 \%$ in patients with FGFR2/HER3 1+ or 2+/0 and FGFR2/HER 3+/1+ or 2+, respectively. When we combined FGFR2 and EGFR expression, no correlation was found in relapse-free survival. FGFR, fibroblast growth factor receptor; HER, human epidermal growth factor receptor; EGFR, EGFR, epidermal growth factor receptor.

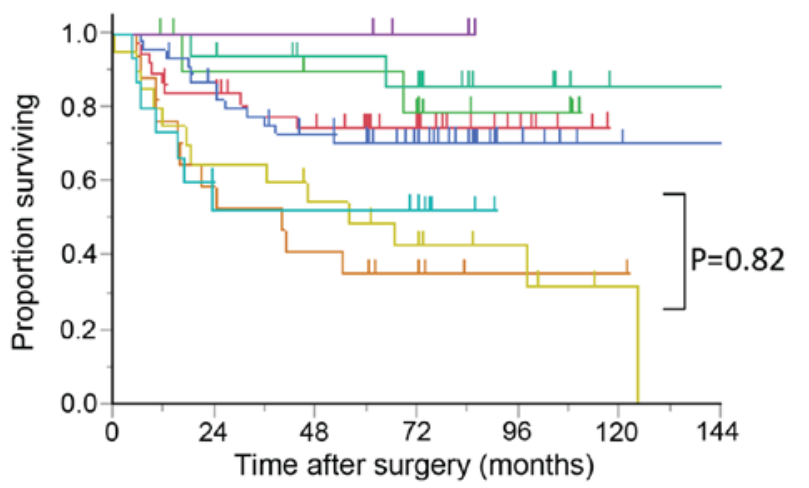

- FGFR2/HER3/EGFR $1+, 2+/ 0 / 1+, 2+$

- FGFR2/HER3/EGFR $1+, 2+/ 0 / 3+$

- FGFR2/HER3/EGFR $1+, 2+/ 1+, 2+/ 1+, 2+$

- FGFR2/HER3/EGFR $1+, 2+/ 1+, 2+/ 3+$

- FGFR2/HER3/EGFR $3+/ 0 / 1+, 2+$

- FGFR2/HER3/EGFR $3+/ 0 / 3+$

- FGFR2/HER3/EGFR $3+/ 1+, 2+/ 1+, 2+$

- FGFR2/HER3/EGFR $3+/ 1+, 2+/ 3+$

Figure 5. Kaplan-Meier curves for relapse-free survival in patients with stage II/III gastric cancer stratified by combination of FGFR2, HER3, and EGFR expressions. Although patients with FGFR2 3+, HER3 1+/2+, and EGFR 3+ belongs to one of the worst survival groups, no significant difference is found between the FGFR2 3+, HER3 1+/2+, and EGFR 3+ group, FGFR2 1+/2+, HER3 1+/2+, and EGFR 3+ group, and FGFR2 3+, HER3 1+/2+, and EGFR 1+/2+ group $(\mathrm{P}=0.82)$. FGFR, fibroblast growth factor receptor; HER, human epidermal growth factor receptor; EGFR, EGFR, epidermal growth factor receptor.

expression was not significantly associated with RFS or OS. When we restricted patients to those with diffuse type cancer, FGFR2 expression levels tended to be negatively correlated with RFS. Moreover, proportion of patients who recurred more than 5 years after surgery was significantly larger in the high FGFR2 expression group than in the low FGFR2 expression group.

The FGFR2 expression level was not significantly associated with RFS or OS. Nagatsuma et al (24) reported that high expression of FGFR 2 correlated with tumor progression and survival in patients with gastric cancer. However, they did not refer to adjuvant chemotherapies and stage distribution. On the other hand, patients in this current study had pathological stage II or III and received adjuvant chemotherapy using S1. Adjuvant S1 chemotherapy might have some power to negate the difference of survival outcomes between different FGFR2 expression levels.

When we restricted patients to those with diffuse type gastric cancer, survival outcome was not significantly but slightly correlated with FGFR2 expression. High FGFR2 expression tended to be slightly worse survival outcomes. Inokuchi et al reported that high FGFR2 expression significantly correlated with tumor progression and survival only in diffuse type gastric cancer (25). If our study patients had not been treated with S1 adjuvant chemotherapy, the negative effect of FGFR2 expression might have significance on survival outcomes.

We previously reported that HER3 expression was a significant independent prognostic factor in patients with stage II/III gastric cancer who receive curative resection and adjuvant chemotherapy with S-1. In our current study, the analyzed patients of which are identical to those of previous one, FGFR2 overexpression augmented prognostic impact of HER3 expression. EGFR family members are reported to be downstream targets of the amplified and highly activated FGFR2 kinase (26). That may explain the augmentation by FGFR 2 expression. On the other hand, among the patients without expression of HER3, patients with high FGFR2 expression had higher survival than those with low FGFR2 expression. However, the difference between these patients is not statistically significant in the log-rank test $(\mathrm{P}=0.19)$. FGFR2 overexpression would not be as strongly associated with RFS as HER3 would be. That may be the reason why high FGFR2 expression did not have significantly lower RFS than low FGFR2 expression in patients without expression of HER3.

An interesting finding of our study was that patients who had recurrence more than 5 years after surgery had a significantly higher probability of having tumors with high FGFR2 
Table IV. Patients who had recurrence $>5$ years after surgery.

\begin{tabular}{cccccccccc}
\hline Age & Sex & Histology & pT & pN & pStage & FGFR2 IHC & HER3 IHC & EGFR IHC & $\begin{array}{c}\text { Interval between } \\
\text { surgery and } \\
\text { recurrence (months) }\end{array}$ \\
\hline 38 & F & Diffuse & $4 \mathrm{a}$ & 0 & IIB & $3+$ & $2+$ & $1+$ & 67 \\
55 & F & Diffuse & $4 \mathrm{a}$ & 0 & IIB & $3+$ & $1+$ & $1+$ & 124 \\
66 & F & Diffuse & $4 \mathrm{a}$ & 1 & IIIA & $3+$ & 0 & $1+$ & 64 \\
63 & M & Diffuse & $4 \mathrm{a}$ & 3 & IIIC & $1+$ & 0 & $3+$ & 68 \\
66 & M & Diffuse & $4 \mathrm{a}$ & 3 & IIIC & $3+$ & $1+$ & $1+$ & 98 \\
\hline
\end{tabular}

FGFR, fibroblast growth factor receptor; HER, human epidermal growth factor receptor; IHC, immunohistochemistry; EGFR, epidermal growth factor receptor; F, female; $\mathrm{M}$, male.

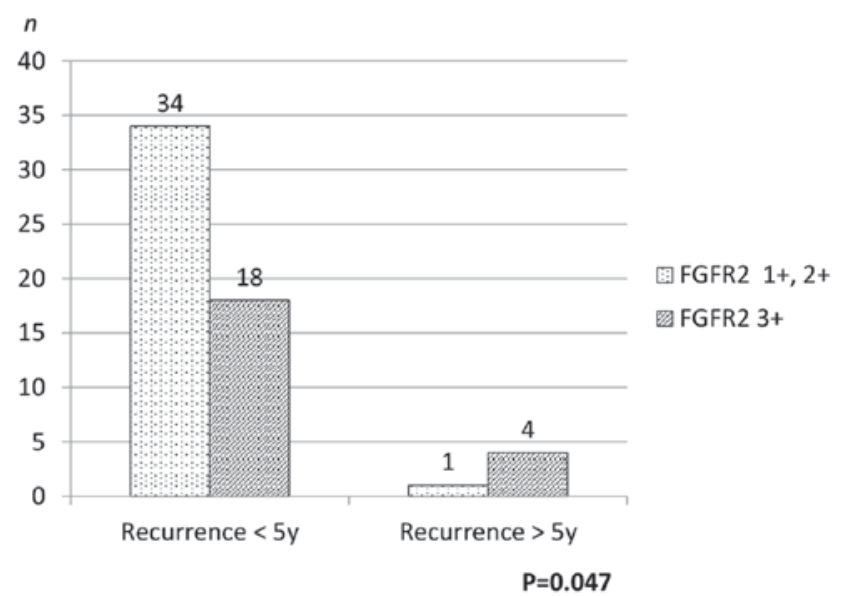

Figure 6. Numbers of patients who recurred less than 5 years after surgery and more than 5 years after surgery per the FGFR2 IHC grade. Proportion of patients who recurred more than 5 years after surgery is significantly larger in the high FGFR2 expression group (FGFR2 3+) than in the low FGFR2 expression group (FGFR2 1+, 2+) [18\% (4/22) vs. $2.9 \%(1 / 35), \mathrm{P}=0.047]$. FGFR, fibroblast growth factor receptor; IHC, immunohistochemistry.

expression. Recurrent sites of long-term failure mostly were peritoneum among diffuse type tumors. The patient with gastric cancer was assumed to be cured at 5 years after surgery unless any signs of recurrence were found. Then, the follow-up was usually terminated. However, we more recently have experienced late recurrence since the introduction of adjuvant chemotherapy using S1. Indeed, $18 \%$ of the recurrent patients with high FGFR2 expression recurred more than 5 years after surgery. This would affect the follow-up strategy for gastrectomized patients who underwent $\mathrm{S} 1$ adjuvant chemotherapy. A few studies have focused on the timing of recurrence and clinicopathological factors (27-29). Preoperative serum carcinoembryonic antigen level, tumor size, LN metastasis and venous invasion have been reported to be independent predictors of the timing of recurrence. However, no biomarker has been reported to be able to predict the timing of recurrence after surgery. To the best of our knowledge, this finding for the first time indicated that FGFR2 could predict long-term failure of postoperative adjuvant S1 chemotherapy in curative advanced gastric cancer. For patients with long-term failure, FGFR2 IHC level should be examined. If FGFR2 overexpression were confirmed, antibodies or small molecule inhibitors targeting FGFR2 might suppress progression of recurrent tumors.

One patient who recurred more than 5 years after surgery had tumors with FGFR2 IHC status of 1+. Instead, this patient had a tumor with EGFR IHC status of 3+. Moreover, EGFR expression level was negatively correlated with FGFR2 expression. This negative correlation might somewhat be associated with the reciprocal relation of the expressions of FGFR2 and EGFR in these patients. We cannot fully understand the underlying mechanism of this phenomenon, but one plausible reason is that both the EGFR and FGFR2-overexpressing cancer cells would have aggressive nature to cause early failure of S1 adjuvant chemotherapy, while either EGFR or FGFR2-overexpressing cancer cells would have relatively slow growing nature and could evade chemotherapy with the capacity of dormancy (30). That might be the reason why the reciprocal expression between the EGFR and the FGFR2 was found in patients who recurred more than 5 years after surgery.

It has been reported that Helicobacter pylori profoundly activates the epidermal growth factor receptor (EGFR) and its family members HER2 and HER3 (31). We only have checked Helicobacter pylori infection in $37(22 \%)$ patients since we did not check it before 2009. In the analysis of these limited patients, no correlations were found between the Helicobacter infection and EGFR, HER3, or FGFR2 expression (data not shown).

Our study has some important limitations. First, the analysis was based on retrospective data collected at a single center, and the number of included patients was so small that we sometimes could not demonstrate statistically significant difference. Second, no rigid rule was applied in terms of dose reduction and termination of S1 administration. Administered dose of $\mathrm{S} 1$ differed between the studied patients. Third, not all the patients were followed up for 10 years, so exact recurrence rate may be different than the observed one.

In conclusion, there was no interaction between FGFR2 expression and patient survival outcomes in patients with stage II/III gastric cancer after the standard treatment in Japan. On the other hand, proportion of patients who recurred more than 5 years after surgery was significantly larger in the high FGFR2 expression group than in the low FGFR2 expression group. Patients with FGFR overexpression in stage II/III 
gastric cancer should be carefully followed-up for more than 5 years after surgery.

\section{References}

1. Torre LA, Bray F, Siegel RL, Ferlay J, Lortet-Tieulent J and Jemal A: Global cancer statistics, 2012. CA Cancer J Clin 65: 87-108, 2015

2. Sakuramoto S, Sasako M, Yamaguchi T, Kinoshita T, Fujii M, Nashimoto A, Furukawa H, Nakajima T, Ohashi Y, Imamura $\mathrm{H}$, et al: Adjuvant chemotherapy for gastric cancer with S-1, an oral fluoropyrimidine. N Engl J Med 357: 1810-1820, 2007.

3. Sasako M, Sakuramoto S, Katai H, Kinoshita T, Furukawa H, Yamaguchi T, Nashimoto A, Fujii M, Nakajima T and Ohashi Y: Five-year outcomes of a randomized phase III trial comparing adjuvant chemotherapy with S-1 versus surgery alone in stage II or III gastric cancer. J Clin Oncol 29: 4387-4393, 2011.

4. Goetz R and Mohammadi M: Exploring mechanisms of FGF signalling through the lens of structural biology. Nat Rev Mol Cell Biol 14: 166-180, 2013

5. Dedes KJ, Wetterskog D, Ashworth A, Kaye SB and Reis-Filho JS: Emerging therapeutic targets in endometrial cancer. Nat Rev Clin Oncol 8: 261-271, 2011.

6. Eswarakumar VP, Lax I and Schlessinger J: Cellular signaling by fibroblast growth factor receptors. Cytokine Growth Factor Rev 16: 139-149, 2005.

7. Turner $\mathrm{N}$ and Grose R: Fibroblast growth factor signalling: From development to cancer. Nat Rev Cancer 10: 116-129, 2010.

8. Brooks AN, Kilgour E and Smith PD: Molecular pathways: Fibroblast growth factor signaling: A new therapeutic opportunity in cancer. Clin Cancer Res 18: 1855-1862, 2012

9. Hattori Y, Odagiri H, Nakatani H, Miyagawa K, Naito K, Sakamoto H, Katoh O, Yoshida T, Sugimura T and Terada M: $\mathrm{K}$-sam, an amplified gene in stomach cancer, is a member of the heparin-binding growth factor receptor genes. Proc Natl Acad Sci USA 87: 5983-5987, 1990.

10. Matsumoto K, Arao T, Hamaguchi T, Shimada Y, Kato K, Oda I Taniguchi H, Koizumi F, Yanagihara K, Sasaki H, et al: FGFR2 gene amplification and clinicopathological features in gastric cancer. Br J Cancer 106: 727-732, 2012.

11. Su X, Zhan P, Gavine PR, Morgan S, Womack C, Ni X, Shen D, Bang YJ, Im SA, Ho Kim W, et al: FGFR2 amplification has prognostic significance in gastric cancer: Results from a large international multicentre study. Br J Cancer 110: 967-975, 2014.

12. Zhao WM, Wang L, Park H, Chhim S, Tanphanich M, Yashiro M and Kim KJ: Monoclonal antibodies to fibroblast growth factor receptor 2 effectively inhibit growth of gastric tumor xenografts. Clin Cancer Res 16: 5750-5758, 2010.

13. Bai A, Meetze K, Vo NY, Kollipara S, Mazsa EK, Winston WM, Weiler S, Poling LL, Chen T, Ismail NS, et al: GP369, an FGFR2-IIIb-specific antibody, exhibits potent antitumor activity against human cancers driven by activated FGFR2 signaling. Cancer Res 70: 7630-7639, 2010

14. Sommer A, Kopitz C, Schatz CA, Nising CF, Mahlert C, Lerchen HG, Stelte-Ludwig B, Hammer S, Greven S, Schuhmacher J, et al: Preclinical efficacy of the auristatin-based antibody-drug conjugate BAY 1187982 for the treatment of FGFR2-positive solid tumors. Cancer Res 76: 6331-6339, 2016.

15. Chang J, Wang S, Zhang Z, Liu X, Wu Z, Geng R, Ge X, Dai C, Liu R, Zhang Q, et al: Multiple receptor tyrosine kinase activation attenuates therapeutic efficacy of the fibroblast growth factor receptor 2 inhibitor AZD4547 in FGFR2 amplified gastric cancer. Oncotarget 6: 2009-2022, 2015.
16. Pearson A, Smyth E, Babina IS, Herrera-Abreu MT, Tarazona N, Peckitt C, Kilgour E, Smith NR, Geh C, Rooney C, et al: High-level clonal FGFR amplification and response to FGFR inhibition in a translational clinical trial. Cancer Discov 6: 838-851, 2016.

17. Kim ST, Jang HL, Lee SJ, Lee J, Choi YL, Kim KM, Cho J, Park SH, Park YS, Lim HY, et al: Pazopanib, a novel multitargeted kinase inhibitor, shows potent in vitro antitumor activity in gastric cancer cell lines with FGFR2 amplification. Mol Cancer Ther 13: 2527-2536, 2014.

18. Xie L, Su X, Zhang L, Yin X, Tang L, Zhang X, Xu Y, Gao Z, Liu K, Zhou M, et al: FGFR2 gene amplification in gastric cancer predicts sensitivity to the selective FGFR inhibitor AZD4547. Clin Cancer Res 19: 2572-2583, 2013.

19. Zhang N, Chang Y, Rios A and An Z: HER3/ErbB3, an emerging cancer therapeutic target. Acta Biochim Biophys Sin (Shanghai) 48: 39-48, 2016.

20. Sithanandam G and Anderson LM: The ERBB3 receptor in cancer and cancer gene therapy. Cancer Gene Ther 15: 413-448, 2008.

21. Yoshioka T, Nishikawa Y, Ito R, Kawamata M, Doi Y, Yamamoto Y, Yoshida M, Omori Y, Kotanagi H, Masuko T and Enomoto K: Significance of integrin $\alpha v \beta 5$ and erbB3 in enhanced cell migration and liver metastasis of colon carcinomas stimulated by hepatocyte-derived heregulin. Cancer Sci 101: 2011-2018, 2010.

22. Ema A, Yamashita K, Ushiku H, Kojo K, Minatani N, Kikuchi M, Mieno H, Moriya H, Hosoda K, Katada N, et al: Immunohistochemical analysis of RTKs expression identified HER3 as a prognostic indicator of gastric cancer. Cancer Sci 105: 1591-1600, 2014

23. Japanese Gastric Cancer Association: Japanese classification of gastric carcinoma-2nd english edition. Gastric Cancer 1: 10-24, 1998.

24. Nagatsuma AK, Aizawa M, Kuwata T, Doi T, Ohtsu A, Fujii H and Ochiai A: Expression profiles of HER2, EGFR, MET and FGFR2 in a large cohort of patients with gastric adenocarcinoma. Gastric Cancer 18: 227-238, 2015.

25. Inokuchi M, Murase H, Otsuki S, Kawano T and Kojima K: Different clinical significance of FGFR1-4 expression between diffuse-type and intestinal-type gastric cancer. World J Surg Oncol 15: 2, 2017.

26. Kunii K, Davis L, Gorenstein J, Hatch H, Yashiro M, Di Bacco A, Elbi C and Lutterbach B: FGFR2-amplified gastric cancer cell lines require FGFR2 and Erbb3 signaling for growth and survival. Cancer Res 68: 2340-2348, 2008.

27. Adachi Y, Oshiro T, Mori M, Maehara Y and Sugimachi K: Prediction of early and late recurrence after curative resection for gastric carcinoma. Cancer 77: 2445-2448, 1996.

28. Eom BW, Yoon H, Ryu KW, Lee JH, Cho SJ, Lee JY, Kim CG, Choi IJ, Lee JS, Kook MC, et al: Predictors of timing and patterns of recurrence after curative resection for gastric cancer. Dig Surg 27: 481-486, 2010.

29. Choi JY, Ha TK and Kwon SJ: Clinicopathologic characteristics of gastric cancer patients according to the timing of the recurrence after curative surgery. J Gastric Cancer 11: 46-54, 2011.

30. Aguirre-Ghiso JA: Models, mechanisms and clinical evidence for cancer dormancy. Nat Rev Cancer 7: 834-846, 2007.

31. Tegtmeyer N, Neddermann M, Asche CI and Backert S: Subversion of host kinases: A key network in cellular signaling hijacked by Helicobacter pylori CagA. Mol Microbiol 105: 358-372, 2017. 